\title{
Model selection in pedestrian detection using multiple kernel learning
}

\author{
Frédéric Suard, Alain Rakotomamonjy, Abdelaziz Bensrhair \\ LITIS EA4051, INSA/Univ de Rouen \\ avenue de l'université, 76800 Saint Etienne du Rouvray, France \\ email: frederic.suard@insa-rouen.fr
}

\begin{abstract}
This paper presents a pedestrian detection method based on the multiple kernel framework. This approach enables us to select and combine different kinds of image representations. The combination is done through a linear combination of kernels, weighted according to the relevance of kernels. After having presented some descriptors and detailed the multiple kernel framework, we propose three different applications concerning combination of representations, automatic parameters setting and feature selection. We then show that the MKL framework enable us to apply a model selection and improve the performance.
\end{abstract}

\section{INTRODUCTION}

Since many years now, pedestrian detection from images has been source of many researches. This topic of research is usually decomposed in two parts : the first one consists in searching for discriminative features while the second part deals with the learning of a decision function from these features.

Recent works [5], [6], [2] have been proposed for representing pedestrian images. For instance, Papageorgiou et al. have used a wavelet decomposition approach for extracting features from images while Shashua et al. have considered histograms of gradients. For both approaches, the underlying objective is to extract from images some discriminative features that help a classifier to recognize images containing or not a pedestrian. These are two examples among the many recent researches that have dealt with the construction of different features.

Once some features have been extracted, the second stage of an automated pedestrian detection algorithm resides in the pedestrian classification problem. During the last years, kernels methods, like Support Vector Machines [9] have shown their efficiency to address such problems [2], [8], [4].

When using SVMs or any other kernel methods, features are integrated into the classifier through a kernel function $\mathbf{k}\left(\mathbf{x}, \mathbf{x}^{\prime}\right)$, where $\mathbf{x}$ and $\mathbf{x}^{\prime}$ represent the features from two different images. In such context, the kernel function acts, in some way, as a measure of similarity between features $\mathbf{x}$ and $\mathbf{x}^{\prime}$ which can be non-vectorial representations.

Recently, a SVM algorithm using multiple kernels have been introduced [3]. The underlying idea of such algorithm is the combination of different heterogenous source of information for learning a decision function. Indeed, this multiple kernel learning (MKL) framework defines the kernel function as a linear combination of kernels :

$$
\mathbf{k}\left(\mathbf{x}, \mathbf{x}^{\prime}\right)=\sum_{k=1}^{K} \beta_{k} \mathbf{k}_{k}\left(\mathbf{x}, \mathbf{x}^{\prime}\right)
$$

where each kernel has been computed from one specific representation of the data. From this equation, it is clear that the idea consists in defining a set of kernels and in combining and selecting a subset of these kernels, each $\beta$ being the weight of a single kernel in the overall combination.

One advantage of the MKL approach resides in the possibility to combine and select the most relevant representations. In fact, the set of kernels can be composed of different kernels built from different types of representations. So instead of using a single representation, one can fuse different representations through the kernel.

One drawback of kernel methods is the need of tuning efficiently the classifier and kernel parameters. Thanks to multiple kernel, it is possible to address this issue by proposing a set of kernels, each of them being computed with a different set of kernel parameters.

Another advantage of multiple kernel is the possibility of selecting only a subset of features. This approach could be assimilated as feature selection, since we can build a set of kernels from each feature and then selecting only the most relevant one by means of the MKL algorithm.

In this paper, we propose to analyze the contribution of multiple kernel learning framework for pedestrian detection. At first, we propose to present the multiple kernel framework and detail the theoretical advantages of such approach. After having highlighted this approach, we propose to combine different kinds of features for classifying pedestrian images. For this purpose, we will present different classical features used in the literature for describing pedestrian and will use multiple kernel approach for combining and retaining the most relevant feature. By doing so, we expect to the algorithm to have a better classification performance due to the feature combination.

We also investigate the contribution of multiple kernel for selecting automatically the kernel parameter and for selecting features.

This paper is organized as follows. In section II, we present the different features that have been extracted from pedestrian images and that have been used afterwards for kernel combination. Then, in section III we detail the multiple 

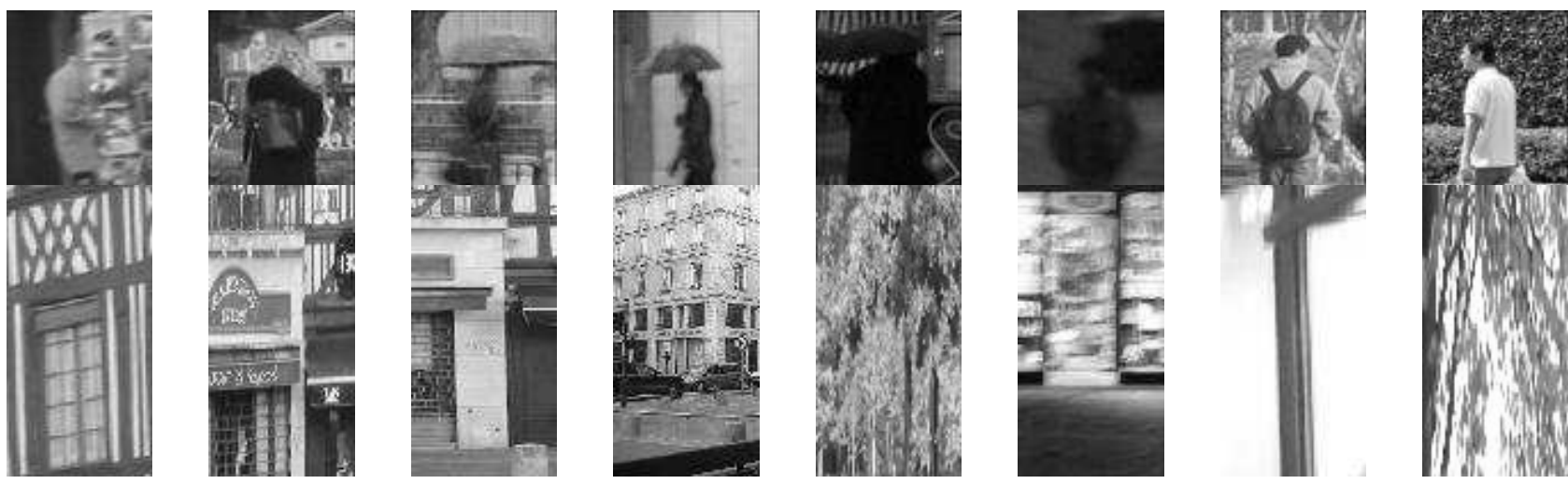

Fig. 3. Examples of pedestrians (first line) and non-pedestrians (second line) manually extracted.

the classifier performance. They stated that the gain in performance can be considerable if different kernels have been obtained from heterogenous sources of information.

The main idea underlying MKL is to consider a new kernel $\mathbf{k}$ as a convex linear combination of other kernels :

$$
\mathbf{k}\left(\mathbf{x}, \mathbf{x}^{\prime}\right)=\sum_{k=1}^{K} \beta_{k} \mathbf{k}_{k}\left(\mathbf{x}, \mathbf{x}^{\prime}\right)
$$

with $\beta_{k} \geq 0, \sum_{k} \beta_{k}=1$ and $\mathbf{k}_{k}(.,$.$) a kernel using a$ single representation using all features composing $x$ or a subset of features. All kernels $\mathbf{k}_{k}$ can also involve different kernel functions such as polynomial or Gaussian kernel using different parameters. Within this framework, the problem of data representation is transferred to the choice of $\beta_{k}$.

The value of coefficients $\alpha, b$ and $\beta$ are obtained by solving the dual of the following optimization problem:

$$
\left\{\begin{aligned}
\min _{\mathbf{w}, \beta, b, \xi} & \frac{1}{2}\left(\sum_{k=1}^{K} \beta_{k}\left\|\mathbf{w}_{k}\right\|_{2}\right)+C \sum_{i=1}^{N} \xi_{i} \\
\text { s.t. } & y_{i} f\left(\mathbf{x}_{i}\right) \geq 1-\xi_{i} \quad \forall i=1, \ldots, N \\
\text { and } & \sum_{k=1}^{K} \beta_{k}=1
\end{aligned}\right.
$$

Bach et al. [1] derived this dual formulation and wrote equivalently :

$$
\left\{\begin{aligned}
\max _{\gamma, \alpha} & \gamma \\
\text { s.t. } & \frac{1}{2} \sum_{i=1}^{N} \sum_{j=1}^{N} \alpha_{i} \alpha_{j} \mathbf{k}_{k}\left(\mathbf{x}_{i}, \mathbf{x}_{j}\right)-\sum_{i=1}^{N} \alpha_{i} \leq \gamma \\
\text { and } & \sum_{i=1}^{N} \alpha_{i} y_{i}=0, \quad \forall i, 0 \leq \alpha_{i} \leq C
\end{aligned}\right.
$$

Hence, finding the optimal solution can be done by solving the following semi-infinite linear program (SILP). We use the formulation of Sonnenburg et al. [7]:

$$
\left\{\begin{aligned}
\max _{\theta, \beta} & \theta \\
\text { s.t. } & \sum_{k=1}^{K} \beta_{k}=1 \\
\text { and } & \sum_{k=1}^{K} \beta_{k} S_{k}(\boldsymbol{\alpha}) \geq \theta
\end{aligned}\right.
$$

with $S_{k}(\boldsymbol{\alpha})=\frac{1}{2} \sum_{i=1}^{N} \sum_{j=1}^{N} \alpha_{i} \alpha_{j} \mathbf{k}_{k}\left(\mathbf{x}_{i}, \mathbf{x}_{j}\right)-\sum_{i=1}^{N} \alpha_{i}$. Sonnenburg et al. proposes to use the Column Generation technique to solve this problem. The idea of the algorithm is to find the optimal value of $\beta$ and $\theta$ for a subset of constraints and determine if $\boldsymbol{\alpha}$ satisfies the constraints $\sum_{k=1}^{K} \beta_{k} S_{k}(\boldsymbol{\alpha}) \geq \theta$. If the constraints are satisfied then the solution is optimal otherwise some constraints are added to the constraints set and the process is continued until convergence of the $\beta$ values is achieved. For each step, a standard SVM solver is used for processing $S_{k}(\boldsymbol{\alpha})$ with an update of the kernel since some constraints and value of $\beta$ can change.

For this paper, we used an optimized version of the algorithm proposed by Sonnenburg et al. [7]. A Matlab code of our implementation is available on demand.

\section{RESUlts}

In this section we will present some results. We then show the interest of the multiple kernel framework approach for combining images representations, for choosing the optimal parameters and for selecting feature.

For this work, we took 310 urban scenes from which we manually extracted 1240 pedestrians and 6220 nonpedestrians. Images were captured during different weather and ligthning conditions : night and day, cloudy and raining weather. Pedestrian are centered and can be partially occluded. Some examples of pedestrians and non-pedestrians extracted are shown on figure 3. All images extracted are rescaled to the same size : $128 \times 64$ pixels.

To compare the results, we plot the rate of true positive against the rate of false positive by counting the number of correct detections and false alarms, when a threshold putted on the prediction value of $f(x)$ (see eq. 1) varies. The AUC is the value of the area under the ROC curve, which should be the nearest of 1 for the best result.

\section{A. Combining representations}

This test consists in combining different representations. We first extracted a set of descriptors for each image of the learning set as presented in section II.

For each type of descriptor, we computed a linear kernel and added all to a set of kernels. We applied the MKL algorithm and retained the most relevant representations.

This last point is simply done by considering the value of coefficients $\beta_{k}$ (see eq. 2). When a coefficient is null, the associated kernel has no influence in the solution, so that the representation is not relevant. On the contrary, when a coefficient value is up to 1 , the representation is very relevant.

We trained the classifier with a learning set containing 500 pedestrians and 500 non-pedestrians, and evaluated this 


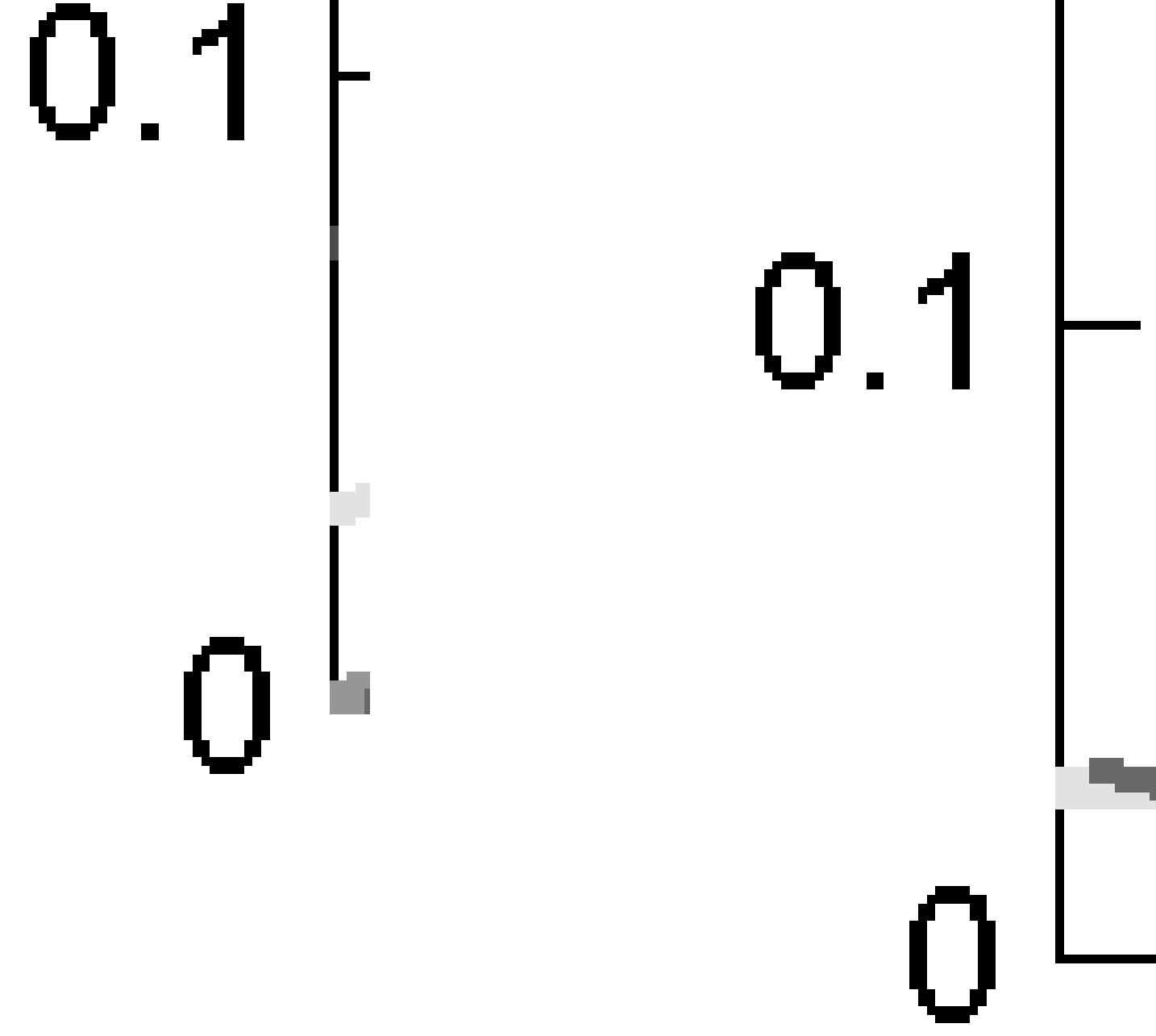

Fig. 4. This figure shows the variation of $\beta$ for each kernel computed from various representations.

On figure 4 we shows the value of $\beta$ for each representation for each iteration. We can note that the $\beta$ corresponding to the representation of HogDalal obtained the largest value, which means that this representation is very relevant compared with other. We also can note that globally the value of $\beta$ does not vary if we consider all test.

The average value of each $\beta$ is then computed :

\begin{tabular}{c|ccccc}
\hline Kernel & Pixel & Gradient & Wavelet & HogShashua & HogDalal \\
\hline$\beta$ & 0.0453 & 0.0571 & 0.0029 & 0 & 0.8947 \\
\hline variance & 0.0115 & 0.0215 & 0.0090 & 0 & 0.0211 \\
\hline
\end{tabular}

We can note that the kernel coming from the HogDalal descriptor has a large weight in the final kernel.

Parrallely, we have improved the performance of each representation separately. The following table shows the average value of AUC obtained.

\begin{tabular}{c|ccccc}
\hline Method & Pixel & Gradient & Wavelet & HogShashua & HogDalal \\
\hline AUC & 0.8806 & 0.8963 & 0.7623 & 0.9592 & 0.9827 \\
\hline$\%$ & 0.8259 & 0.8171 & 0.7237 & 0.8919 & 0.9399 \\
\hline
\end{tabular}

Using the MKL approach, we obtained an AUC of 0.9859 and a good recognition rate of $0.9472 \%$, which performs better than any single representations. We can also notice that some descriptors are more relevant than other, in particular, using histograms of oriented gradient with a cutting proposed by Dalal reveals to be very efficient,
Fig. 5. This figure shows the variation of $\beta$ for each kernel computed from various representations.

We also plot all values of $\beta$ obtained for each test on figure 5 . We can see that the value of most relevant presentation is lower than the value of the coefficient HOGDalal. Moreover, the variance for all coefficients is larger, since all kernels participate to the solution.

We can notice a relation between the HogShashua and the Gradient information. This relation can be explain by the fact that these descriptors involve the same type of information but the HogShashua exploits only a subpart of the image. When all the pedestrians are well centered this descriptors reveals to be more efficient. On the contrary, the gradient information is more relevant.

So we can conclude that the MKL approach can select the most relevant representation, but is also capable to avoid the redundancy of information. A combination of representations also improves the global performance. 


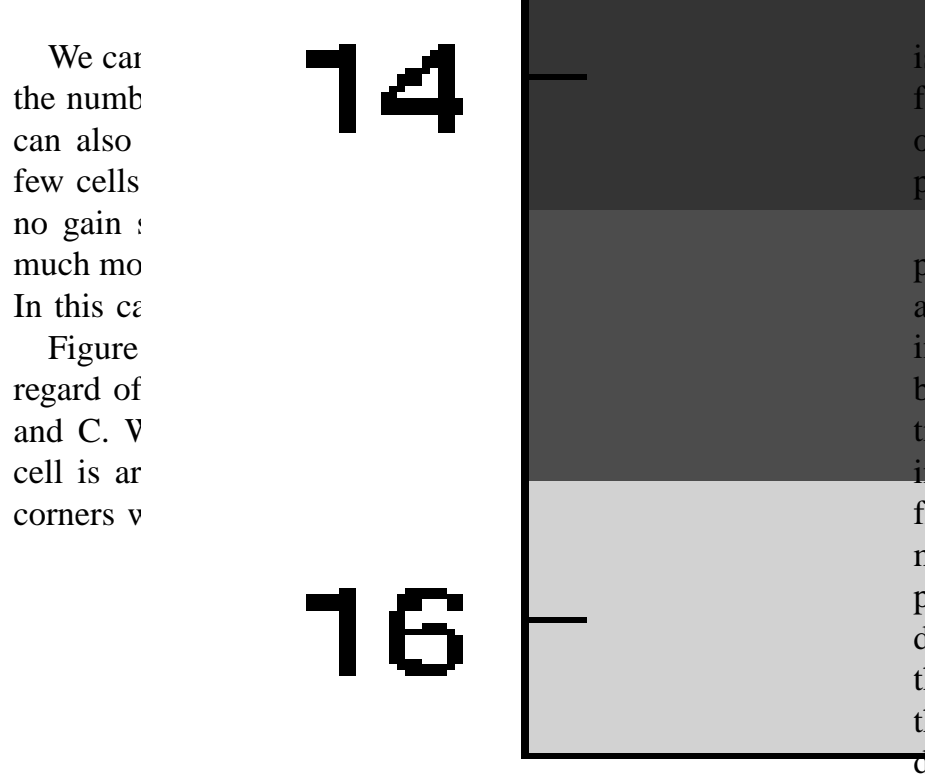

feature selection, when a kernel is computed for each eature of a descriptor. Using MKL enabled us to select only the most relevant features and to improve the global erformance. paper is that among all the
we have used the HOGDalal
ost efficient. However, it is
esentations are in some sense
Hence one of our perspec-
sentations based on different
spectives based on the MKL
First we plan to test deeply
ne all tests described in this
e different features of various
heters and type of kernels. For
multiple kernel is the size of
on the size of the learning dataset and une number or kernels. Constaering the high memory necessary to compute large kernel sets $(>500)$ we are looking now how to deal with such large scale multiple kernel.

\section{REFERENCES}

Fig. 7. This figure shows the value of $\beta$, each kernel of the kernel set corresponding to a single cell of the image for parameter set A (left) and $\mathrm{C}$ (right).

A last advantage of using a MKL approach to select the most relevant features resides in the complexity of the process. Classical techniques, like forward-backward, are suppose to iterate the same processus a large number of time to select the most relevant features. We have to compute all data and the associated kernel to remove or add only 1 feature at a time. With a MKL approach all we have to do is to build one kernel per feature and the algorithm will note the relevance of each kernel by means of the coefficient $\beta$.

\section{CONCLUSION AND PERSPECTIVES}

We have presented a novel approach for model selection using multiple kernel. The idea consists in defining a new kernel as a linear combination of various kernels. The combination is weighted by some coefficients with regard of the kernel relevance. After having introduced some standard descriptors used for pedestrian detection, we have presented in detail the multiple kernel framework. Then, we have proposed to apply multiple kernel for pedestrian detection with three different applications.

First we used MKL to combine and select different representations of images, each kernel of the set corresponding to a single representation. This approach enable us to select the more relevant application and improved the detection performance. The second application was due to automatic setting. One drawback of classical kernel machine resides in the kernel setting. Thanks to multiple kernel, we showed that it is possible to set automatically the kernel, by computing a set of kernels with different paramters. The last point

[1] Francis R. Bach, Gert R. G. Lanckriet, and Michael I. Jordan. Multiple kernel learning, conic duality, and the smo algorithm. In ICML '04: Proceedings of the twenty-first international conference on Machine learning, page 6, 2004.

[2] Navneet Dalal and Bill Triggs. Histograms of oriented gradients for human detection. In Cordelia Schmid, Stefano Soatto, and Carlo Tomasi, editors, International Conference on Computer Vision and Pattern Recognition, volume 2, pages 886-893, June 2005.

[3] Gert R. G. Lanckriet, Nello Cristianini, Peter Bartlett, Laurent El Ghaoui, and Michael I. Jordan. Learning the kernel matrix with semidefinite programming. J. Mach. Learn. Res., 5:27-72, 2004.

[4] Stefan Munder and Dariu Gavrila. An experimental study on pedestrian classification. IEEE Transactions on Pattern Analysis and Machine Intelligence, 28-11:1863-1868, 2006.

[5] Constantine Papageorgiou and Tomaso Poggio. Trainable pedestrian detection. In Proceedings of the 1999 International Conference on Image Processing, pages 35-39, 1999.

[6] Amnon Shashua, Yoram Gdalyahu, and Gaby Hayon. Pedestrian detection for driving assistance systems: Single-frame classification and system level performance. In Proceedings of IEEE Intelligent Vehicles Symposium, 2004.

[7] Sren Sonnenburg, Gunnar Raetsch, and Christin Schaefer. A general and efficient multiple kernel learning algorithm. In Advances in Neural Information Processing Systems 18, pages 1273-1280, 2005.

[8] Frédéric Suard, Alain Rakotomamonjy, Abdelaziz Bensrhair, and Alberto Broggi. Pedestrian detection using infrared images and histograms of oriented gradients. In Intelligent Vehicles Symposium, Tokyo, Japan, pages 206-212, June 2006.

[9] Vladimir Vapnik. The Nature of Statistical Learning Theory. Springer, N.Y, 1995.

[10] Fengliang Xu, Xia Liu, and Kikuo Fujimura. Pedestrian detection and tracking with night vision. IEEE Transactions on Intelligent Transportation Systems, 6-1:63-71, march 2005. 